\title{
The Finite Element Method with Penalty
}

\author{
By Ivo Babuška*
}

\begin{abstract}
An application of the penalty method to the finite element method is analyzed. For a model Poisson equation with homogeneous Dirichlet boundary conditions, a variational principle with penalty is discussed. This principle leads to the solution of the Poisson equation by using functions that do not satisfy the boundary condition. The rate of convergence is discussed.
\end{abstract}

1. Introduction. The finite element method in all of its versions has become the subject of current practical and theoretical study. A particular problem associated with the finite element method has recently attracted considerable interest. Specifically, this problem is the application of variational principles to spaces of functions in which the boundary conditions need not be satisfied. See for example references [1] to [7].

In references [5] and [6], this author has studied the penalty method approach to this problem. This approach consists in the use of a "penalty" parameter which depends on the smoothness of the original problem. The selection of the penalty parameter is, in some sense, arbitrary. Moreover, the solution of the original problem may be quite sensitive to this parameter.

This paper studies the model Poisson problem $-\Delta u=f$ with homogeneous boundary conditions of Dirichlet type. A variational principle for this model problem on spaces of functions not satisfying the boundary conditions is studied and, based on this principle, a variant of the finite element method is given. This new scheme has a rate of convergence that is arbitrarily close to the optimal rate found by using the usual finite element method with elements satisfying the boundary conditions. The analysis also shows that the finite element method with penalty is not overly sensitive to the choice of the penalty parameter.

2. Some Principal Notions. Let $R_{n}$ be an $n$-dimensional Euclidian space. For $x \equiv\left(x_{1}, \cdots, x_{n}\right) \in R_{n}$, we define $\|x\|^{2}=\sum_{i=1}^{n} x_{i}^{2}$ and $d x=d x_{1} \cdots d x_{n}$.

Let $\Omega$ be a bounded domain in $R_{n}$ with boundary $\Gamma \in C^{\infty}$.

Let $H^{m}\left(R_{n}\right), H^{m}(\Omega)$ and $H^{m}(\Gamma), m \geqq 0, m$ not necessarily an integer, be the fractional Sobolev spaces of order $m$ on $R_{n}, \Omega$ and $\Gamma$, respectively. We will designate the respective norms of these Sobolev spaces by $\|\cdot\|_{H^{m}\left(R_{n}\right)},\|\cdot\|_{H^{m}(\Omega)}$ and $\|\cdot\|_{H^{m}(\Gamma)}$. Recall that $H^{m}(\Omega)$ and $H^{m}(\Gamma)$ are sometimes also denoted by $W_{2}^{m}(\Omega)$ and $W_{2}^{m}(\Gamma)$, respectively, and that $H^{0}(\Omega)=L_{2}(\Omega)$ and $H^{0}(\Gamma)=L_{2}(\Gamma)$. Let the spaces $H_{0}^{m}(\Omega)$ be the closure in the $H^{m}(\Omega)$ norm of the functions in $H^{m}(\Omega)$ which have compact support in $\Omega$.

Received November 24, 1971.

AMS (MOS) subject classifications (1970). Primary 65N15, 65N30; Secondary 35J05.

Key words and phrases. Numerical solution of PDE, elliptic equations, finite element method, penalty method.

* This research was supported by the Atomic Energy Commission under contract number AEC AT (40-1) 3443. 
Throughout the entire paper, $C$ will denote a generic constant with different values in different places. Similarly, $\epsilon$ will denote an arbitrary positive real number with different values in different places.

Now let us introduce the notion of a $(t, k)$-regular system of functions. Let $0<$ $h \leqq 1$ and $0 \leqq k \leqq t$. A linear system of functions $g \in H^{k}\left(R_{m}\right)$ will be called $(t, k)$ regular and will be denoted by $\gamma_{h}^{\ell, k}\left(R_{m}\right)$ if and only if

(i) for every $h$ and every $w \in H^{l}\left(R_{m}\right)$, there exists a function $g \in \gamma_{h}^{t, k}\left(R_{n}\right)$ such that, for any $s \leqq l, 0 \leqq s \leqq k$,

$$
\|w-g\|_{H^{*}\left(R_{n}\right)} \leqq C h^{\mu}\|w\|_{H^{l}\left(R_{n}\right)},
$$

where $C$ is independent of $s, h$ and $w$ and where $\mu=\min (l-s, t-s)$; and

(ii) if $w \in H^{l}\left(R_{n}\right)$ has a compact support $S$, then $g$ in the inequality (2.1) has a compact support $S_{\lambda}^{h}$ such that

$$
S_{\lambda}^{h} \subset\left\{x \in R_{n} \mid d(x, S) \leqq \lambda h\right\},
$$

where $d(x, S)$ is the distance from $x$ to $S$ and $\lambda$ is a positive real number independent of $S, s, h$ and $w$.

By $\gamma_{h}^{t, k}(\Omega)$, we denote the set of all $g \in \gamma_{h}^{t, k}\left(R_{n}\right)$ being restricted to $\Omega$.

In [9], a special $(t, k)$-regular system was studied. This system is defined as the totality of all functions of the form

$$
\sum_{j=1}^{r} \sum_{p} c(p, j) \omega_{i}(x / h-p)
$$

where $p \equiv\left(p_{1}, \cdots, p_{n}\right), p_{i}$ is an integer and the $\omega_{i} \in H^{k}\left(R_{n}\right)$ are fixed functions with compact support which satisfy certain conditions as explained in [9].

As a model problem, in this paper we will be interested in solving Poisson's equation

$$
-\Delta u=f \text { on } \Omega,
$$

with the homogeneous Dirichlet boundary condition

$$
u=0 \text { on } \Gamma \text {. }
$$

We will seek a weak solution to the problem, i.e. a function $u \in H_{0}^{1}(\Omega)$ such that, for every $v \in H_{0}^{1}(\Omega)$,

$$
B(u, v)=(f, v)
$$

where

$$
B(u, v)=\int_{\Omega}\left(\sum_{i=1}^{n} \frac{\partial u}{\partial x_{i}} \frac{\partial v}{\partial x_{i}}\right) d x
$$

and

$$
(f, v)=\int_{\Omega} f v d x .
$$

It is well known that for $f \in L_{2}(\Omega)$ there exists exactly one weak solution of the problem. Furthermore, it is known (see [8, p. 203]) that $f \in H^{m}(\Omega)$ implies $u \in H^{m+2}(\Omega)$ and $\|u\|_{H^{m+2}(\Omega)} \leqq C\|f\|_{H^{m}(\Omega)}$, where $C$ does not depend on $f$. 
Let us remark that the solution $u$ of (2.5) coincides with the function which minimizes the quadratic functional

$$
F(v)=B(v, v)-2(f, v)
$$

over $H_{0}^{1}(\Omega)$.

3. The Finite Element Method With Penalty. Error estimates in $H^{1}(\Omega)$. In expression (2.8), it is crucial that we minimize $F(v)$ not over $H^{1}(\Omega)$, but only over $H_{0}^{1}(\Omega)$, i.e. over the space of functions in $H^{1}(\Omega)$ which vanish on $\Gamma$. The penalty method avoids this restriction. This is important from the computational point of view since the construction of functions vanishing on $\Gamma$ is often technically complicated.

Let $u_{0}$ be the weak solution of our Poisson problem (2.3) and (2.4). Let us construct an approximate solution $v_{h} \in \bar{\gamma}_{h}^{t, k}(\Omega), k \geqq 1$, with $\bar{\gamma}_{h}^{t, k}(\Omega)$ the closure of $\gamma_{h}^{t, k}$ in $H^{1}(\Omega)$ such that the function $v_{h}$ minimizes the quadratic functional

$$
F_{\sigma}(v)=B(v, v)+h^{-\sigma}\langle v, v\rangle-2(f, v), \quad \sigma>0,
$$

over $v \in \bar{\gamma}_{h}^{t, k}(\Omega),{ }^{* *}$ where we denote

$$
\langle v, v\rangle=\oint_{\Gamma} v^{2} d s
$$

and $B(v, v)$ and $(f, v)$ are defined by expressions (2.6) and (2.7). The function $v_{h}$ is uniquely determined and, clearly, depends on the choice of the parameter $\sigma$ (and, of course, on $\gamma_{h}^{t, k}(\Omega)$ ). To emphasize this fact, we will write $v_{\sigma, h}$ instead of $v_{h}$. In [5] and [6], the author proved the following theorem.***

THEOREM 3.1. Let $f \in H^{l}(\Omega), l \geqq 0$. Let $u_{0}$ be the solution of the problem (2.3), (2.4) and let $v_{\sigma, h} \in \gamma_{h}^{t, k}(\Omega), k \geqq 1$, be the approximate solution introduced above. Then

$$
\left\|u_{0}-v_{\sigma, h}\right\|_{H^{1}(\Omega)} \leqq C(\epsilon) h^{\mu-\epsilon}\|f\|_{H^{l}(\Omega)},
$$

where $\epsilon>0$ is arbitrary, $C(\epsilon)$ is independent of $f$ and $h$, and

$$
\mu=\min \left[l+1, l+\frac{3}{2}-\frac{1}{2} \sigma, \frac{1}{2} \sigma, t-1, t-\frac{1}{2}-\frac{1}{2} \sigma\right] .
$$

Let us discuss the theorem. It can be shown that the first of the four terms in (3.3) cannot be improved. $\dagger$ Taking $t \geqq l+2$ and choosing an optimal $\sigma$, namely $\sigma=l+\frac{3}{2}$, we obtain a rate of convergence $\mu=\frac{1}{2} l+\frac{3}{4}$. This rate is substantially less than the maximally possible one, namely $l+1$. The second important disadvantage is that overestimating the parameter $\sigma$ with respect to $l$ may endanger the convergence. This behavior implies that the penalty method will very likely be sensitive to the choice of the parameter $\sigma$.

Some numerical experiments have suggested that the method actually behaves better than this theorem indicates. Let us now show that the theorem may be substantially improved.

We first prove a lemma.

** Let us remark that the expression $B(u, u)+h^{-\sigma}\langle u, u\rangle$ is equivalent to $\|u\|^{2}$.

*** Theorem 3.1 is stated in more general form in [6]. A very similar theorem is proved in [3].

$\dagger$ See reference [10]. 
LEMMA 3.1. Let $w \in H^{l}(\Omega)$, where $l$ is an integer, $l \geqq 2$, and let $w=0$ on $\Gamma$. Then there exists a function $g \in \gamma_{h}^{t, k}(\Omega), t \geqq l, k \geqq 1$, such that, for $\sigma>0$,

$$
\|w-g\|_{H^{1}(\Omega)}^{2}+h^{-\sigma}\left\|\frac{\partial w}{\partial n} h^{\sigma}+g\right\|_{H^{\circ}(\Gamma)}^{2} \leqq C(\epsilon) h^{2 \mu-\epsilon}\|w\|_{H^{l}(\Omega)}^{2},
$$

where $\epsilon>0$ is arbitrary, $C(\epsilon)$ depends only on $\epsilon$ and not on $w$ and $h$, and

$$
\mu=\min \left[\frac{t-x}{t-1}(l-1), \sigma, \frac{\sigma}{2}+l-\frac{3}{2}\right]
$$

with $x=\max (1,(\sigma+1) / 2)$. and $\dagger \dagger$

Proof. (1) By assumption, $w \in H^{l}(\Omega), l \geqq 2$. Therefore, $\partial w / \partial n \in H^{l-3 / 2}(\Gamma)$

$$
\|\partial w / \partial n\|_{H^{l-3 / 2(\Gamma)}} \leqq C\|w\|_{H^{l}(\Omega)} .
$$

Define $v=\partial w / \partial n$ on $\Gamma$. Then there exists a harmonic function $V \in H^{l-1}(\Omega)$ such that $V=v$ on $\Gamma$ and such that $\dagger \dagger$

$$
\begin{aligned}
\|V\|_{H^{l-1}(\Omega)} & \leqq C\|v\|_{H^{l-3 / 2}(\Gamma)} \\
& \leqq C\|w\|_{H^{l}(\Omega)} .
\end{aligned}
$$

(2) Using Theorem 3.1 of [11], there exists a function $\varphi \in \gamma_{h}^{t, k}(\Omega), t \geqq k \geqq s$, $l-1 \geqq s \geqq 0$, such that

$$
\|V-\varphi\|_{H^{*}(\Omega)} \leqq C h^{\mu}\|V\|_{H^{l-1}(\Omega)},
$$

where $\mu=\min [t-s, l-1-s]$. Combining inequalities (3.7) and (3.8), we obtain

$$
\|V-\varphi\|_{H^{\circ}(\Omega)} \leqq C h^{\mu}\|f\|_{H^{l}(\Omega)} .
$$

Given $\epsilon>0$, taking $s=\frac{1}{2}+\epsilon$ and using the embedding theorem, we obtain

$$
\|V-\varphi\|_{H^{\circ}(\Gamma)} \leqq C(\epsilon) h^{l-3 / 2-\epsilon}\|f\|_{H^{l}(\Omega)} .
$$

(3) By Theorem 4.4 of [11], for $t \geqq l, k \geqq 1$, there exists $\xi \in \gamma_{h}^{t, k}(\Omega)$ such that

$$
\|w-\xi\|_{H^{1}(\Omega)}+h^{-\sigma / 2}\|\xi\|_{H^{\circ}(\Gamma)} \leqq C(\epsilon) h^{\alpha-\epsilon}\|w\|_{H^{l}(\Omega)},
$$

where $\epsilon>0$ is arbitrary,

$$
\kappa=\frac{t-x}{t-1}(l-1)
$$

and

$$
x=\max \left(1, \frac{1}{2}+\frac{1}{2} \sigma\right) .
$$

(4) For $t \geqq l \geqq 2$ and $k \geqq 1$, taking $g=\xi-h^{\sigma} \varphi \in \gamma_{h}^{t, k}(\Omega)$ and using inequalities (3.10) and (3.11), we obtain

$\dagger \dagger$ For a proof of this, see Theorem 9.4 of [8].

$\dagger+\dagger$ See [8, p. 203]. 


$$
\begin{aligned}
\|w-g\|_{H^{1}(\Omega)}^{2}+ & h^{-\sigma}\left\|\frac{\partial w}{\partial n} h^{\sigma}+g\right\|_{H^{\circ}(\Gamma)}^{2} \\
= & \left\|w-\xi+V h^{\sigma}-h^{\sigma}(V-\varphi)\right\|_{H^{1}(\Omega)}^{2} \\
& +h^{-\sigma}\left\|\frac{\partial w}{\partial n} h^{\sigma}-V h^{\sigma}+h^{\sigma}(V-\varphi)+\xi\right\|_{H^{\circ}(\Gamma)}^{2} \\
\leqq & C\left[\|w-\xi\|_{H^{1}(\Omega)}^{2}+h^{2 \sigma}\|V-\varphi\|_{H^{\circ}(\Omega)}^{2}\right. \\
& \left.\quad+h^{2 \sigma}\|V\|_{H^{1}(\Omega)}^{2}+h^{-\sigma}\|\xi\|_{H^{\circ}(\Gamma)}^{2}+h^{\sigma}\|V-\varphi\|_{H^{\circ}(\Gamma)}^{2}\right] \\
\leqq & C(\epsilon) h^{2 r-\epsilon}\|w\|_{H^{l}(\Omega)}^{2},
\end{aligned}
$$

where $\epsilon>0$ is arbitrary and

$$
\tau=\min \left[\frac{t-x}{t-1}(l-1), \sigma, t-1+\sigma, l-2+\sigma, l-\frac{3}{2}+\frac{\sigma}{2}\right]
$$

with

$$
x=\max \left(1, \frac{1}{2}(\sigma+1)\right) .
$$

Recalling the assumption that $t \geqq l \geqq 2$, we obtain inequality (3.4) and the proof of the lemma is complete.

We can now prove the improved version of Theorem 3.1.

THEOREM 3.2. Let $f \in H^{l}(\Omega)$, where l is a nonnegative integer. Let $u_{0}$ be the weak solution of the problem (2.3), (2.4) and let $v_{\sigma, h} \in \bar{\gamma}_{h}^{t, k}(\Omega), k \geqq 1, t \geqq l+2, \sigma>0$, be the approximate solution of the problem. Then

$$
\left\|u_{0}-v_{\sigma, h}\right\|_{H^{1}(\Omega)} \leqq C(\epsilon) h^{\mu-\epsilon}\|f\|_{H^{l}(\Omega)},
$$

where $\epsilon>0$ is arbitrary, $C(\epsilon)$ is independent of $f$ and $h$ and

$$
\mu=\min \left(\sigma, \frac{\sigma+1}{2}+l, \frac{t-x}{t-1}(l+1)\right)
$$

with

$$
x=\max \left(1, \frac{1}{2}(\sigma+1)\right) .
$$

Proof. Let us define a quadratic functional $R_{\sigma}(v), v \in H^{1}(\Omega)$, similar to expression (3.1):

$$
R_{\sigma}(v)=B\left(u_{0}-v, u_{0}-v\right)+h^{-\sigma}\left\langle\frac{\partial u_{0}}{\partial n} h^{\sigma}+v, \frac{\partial u_{0}}{\partial n} h^{\sigma}+v\right\rangle .
$$

Taking into account that $u_{0}$ is the weak solution of the problem (2.3), (2.4), it is easy to show that

$$
R_{\sigma}(v)=K_{\sigma}\left(u_{0}\right)+F_{\sigma}(v),
$$

where $F_{\sigma}(v)$ is defined by expression (3.1) and

$$
K_{\sigma}\left(u_{0}\right)=B\left(u_{0}, u_{0}\right)+h^{\sigma}\left\langle\partial u_{0} / \partial n, \partial u_{0} / \partial n\right\rangle
$$


Since $f \in H^{l}(\Omega), l \geqq 0$, then $u_{0} \in H^{l+2}(\Omega)$ and we have $\partial u_{0} / \partial n \in H^{l+1 / 2}(\Gamma)$. Therefore, expressions (3.20) and (3.22) both make sense. The functional $K_{\sigma}\left(u_{0}\right)$ does not depend upon $\boldsymbol{v}$. Therefore, the minimizations of expression (3.1) and expression (3.20) both lead to the same element $v_{\sigma, h}$.

Using Lemma 3.1, we see that there exists a function $g \in \gamma_{h}^{t, k}(\Omega)$ such that

$$
R_{\sigma}(g) \leqq C(\epsilon) h^{2 \mu-\epsilon}\|f\|_{H^{l}(\Omega)}^{2},
$$

where $\epsilon>0, C(\epsilon)$ is independent of $h$ and $f$, and

$$
\mu=\min \left[\frac{t-x}{t-1}(l+1), \sigma, \frac{\sigma}{2}+l+\frac{1}{2}\right] .
$$

From inequality (3.23) and the definition of $v_{\sigma, h}$, we obtain

$$
R_{\sigma}\left(v_{\sigma, h}\right) \leqq C(\epsilon) h^{2 \mu-\epsilon}\|f\|_{H^{l}(\Omega)},
$$

where $\mu$ is given by expression (3.24). Therefore,

$$
B\left(u_{0}-v_{\sigma, h}, u_{0}-v_{\sigma, h}\right) \leqq C(\epsilon) h^{2 \mu-\epsilon}\|f\|_{H^{l}(\Omega)}^{2}
$$

and

$$
\begin{aligned}
\left\langle v_{\sigma, h}, v_{\sigma, h}\right\rangle & \leqq C h^{2 \mu+\sigma-\epsilon}\|f\|_{H^{l}(\Omega)}^{2}+C h^{2 \sigma}\left\langle\frac{\partial u_{0}}{\partial n}, \frac{\partial u_{0}}{\partial n}\right\rangle \\
& \leqq C\left[h^{2 \mu+\sigma-\epsilon}+h^{2 \sigma}\right]\|f\|_{H^{l}(\Omega)}^{2} .
\end{aligned}
$$

From

$$
\left\|u_{0}-v_{\sigma, h}\right\|_{H^{1}(\Omega)}^{2} \leqq C\left[B\left(u_{0}-v_{\sigma, h}, u_{0}-v_{\sigma, h}\right)+\left\langle v_{\sigma, h}, v_{\sigma, h}\right\rangle\right],
$$

we obtain

$$
\left\|u_{0}-v_{\sigma, h}\right\|_{H^{1}(\Omega)} \leqq C h^{k-\epsilon}\|f\|_{H^{l}(\Omega)},
$$

where

$$
\kappa=\min [\sigma, \mu]=\mu .
$$

This completes the proof of Theorem 3.2 for $l$ a nonnegative integer and $t \geqq l+2$.

By using basic theorems about interpolated Sobolev spaces, we can easily generalize the theorem to cover the case of $l$ being any nonnegative real number.

Let us now compare Theorem 3.1 to Theorem 3.2. The advantages of Theorem 3.2 over 3.1 are as follows:

(i) For $\sigma<t$, convergence is independent of the value of $l$.

(ii) For $t$ sufficiently large, we can select $\sigma$ so that $\mu=l+1-\epsilon$.

(iii) From (i), we see that the error is not too sensitive to the changes in $\sigma$.

4. The Error Estimate in $L_{2}(\Omega)$. Let us now derive the error estimate in the space $L_{2}(\Omega)$. The main idea of the proof is similar to the proof of the error bounds in the finite element method without penalty.

THEOREM 4.1. Let $f \in H^{l}(\Omega)$, where l is a nonnegative integer, and let $u_{0}$ be the weak solution of the problem (2.3), (2.4). Let $\sigma \geqq 0$. For $t \geqq l+2$ and $k \geqq 1$, let $v_{\sigma, h} \in \gamma_{h}^{t, k}(\Omega)$ be the approximate solution introduced in Section 3. Then

$$
\left\|u_{0}-v_{\sigma, h}\right\|_{L_{2}(\Omega)} \leqq C(\epsilon) h^{r-\epsilon}\|f\|_{H^{l}(\Omega)},
$$


where $\epsilon>0, C(\epsilon)$ does not depend on $h$ and $f$ and

$$
\tau=\min \left[\kappa+\mu, \kappa+\frac{1}{2} \sigma, \mu+\frac{1}{2} \sigma, \sigma\right]
$$

with $\mu$ given by expression (3.18), and $\kappa$ given by

$$
\kappa=\frac{t-x}{t-1} \text { and } x=\max \left(1, \frac{\sigma+1}{2}\right) .
$$

Proof. (1) Let us denote the error by $\epsilon_{h}=u_{0}-u_{\sigma, h}$. In Theorem 3.2, we proved that

$$
\left\|\epsilon_{h}\right\|_{H^{1}(\Omega)} \leqq C(\epsilon) h^{\mu-\epsilon}\|f\|_{H^{l}(\Omega)},
$$

where $\mu$ was given by expression (3.18). Therefore, we also have

$$
\left\|\epsilon_{h}\right\|_{L_{2}(\Omega)} \leqq C(\epsilon) h^{\mu-\epsilon}\|f\|_{H^{l}(\Omega)} .
$$

Denote by $V_{h} \in H_{0}^{1}(\Omega)$ a function such that

$$
B\left(V_{h}, v\right)=\left(\epsilon_{h}, v\right)
$$

for all $v \in H_{0}^{1}(\Omega)$. Then $V_{h}$ is the weak solution of the problem (2.3), (2.4) with $f=\epsilon_{h}$. Therefore,

$$
\left\|V_{h}\right\|_{H^{2}(\Omega)} \leqq C\left\|\epsilon_{h}\right\|_{L_{2}(\Omega)} .
$$

(2) The approximate solution $v_{\sigma, h}$ minimizes expression (3.20). Therefore, for every $v \in \bar{\gamma}_{h}^{t, k}(\Omega)$, we have

$$
B\left(\epsilon_{h}, v\right)+h^{-\sigma}\left\langle\frac{\partial u_{0}}{\partial n} h^{\sigma}-\epsilon_{h}, v\right\rangle=0 .
$$

By Theorem 4.4 of [11] for $t \geqq 2$ and $k \geqq 1$, there exists a function $g_{h} \in \gamma_{h}^{t, k}(\Omega)$ such that

$$
\begin{aligned}
& \left\|V_{h}-g_{h}\right\|_{H^{1}(\Omega)}+h^{-\sigma / 2}\left\|g_{h}\right\|_{H^{\circ}(\Gamma)} \\
& \leqq C(\epsilon) h^{\kappa-\epsilon}\left\|V_{h}\right\|_{H^{2}(\Omega)} \leqq C(\epsilon) h^{\kappa-\epsilon}\left\|\epsilon_{h}\right\|_{L_{2}(\Omega)},
\end{aligned}
$$

where $\kappa=(t-x) /(t-1)$ and $x=\max \left(1, \frac{1}{2}(\sigma+1)\right)$.

(3) It is easy to see that

$$
B\left(\epsilon_{h}, V_{h}\right)=\left(\epsilon_{h}, \epsilon_{h}\right)+\left\langle\epsilon_{h}, \partial V_{h} / \partial n\right\rangle
$$

By using Eq. (4.5) with $v=g_{h}$, we obtain

$$
B\left(\epsilon_{h}, V_{h}\right)+B\left(\epsilon_{h}, g_{h}-V_{h}\right)=h^{-\sigma}\left\langle\epsilon_{h}, g_{h}\right\rangle-\left\langle\partial u_{0} / \partial n, g_{h}\right\rangle .
$$

Hence, by Eq. (4.7),

$$
\begin{aligned}
\left\|\epsilon_{h}\right\|_{L_{2}(\Omega)}^{2} \leqq & \left|B\left(\epsilon_{h}, g_{h}-V_{h}\right)\right|+h^{-\sigma}\left|\left\langle\epsilon_{h}, g_{h}\right\rangle\right| \\
& +\left|\left\langle\epsilon_{h}, \partial V_{h} / \partial n\right\rangle\right|+\left|\left\langle\partial n_{0} / \partial n, g_{h}\right\rangle\right|
\end{aligned}
$$

Furthermore, we have

$$
\begin{aligned}
\left|B\left(\epsilon_{h}, g_{h}-V_{h}\right)\right| & \leqq\left\|\epsilon_{h}\right\|_{H^{1}(\Omega)}\left\|g_{h}-V_{h}\right\|_{H^{1}(\Omega)} \\
& \leqq C h^{\mu+\kappa-\epsilon}\|f\|_{H^{l}(\Omega)}\left\|\epsilon_{h}\right\|_{L_{2}(\Omega)}
\end{aligned}
$$


Using inequalities (3.27) and (4.6) and the fact that $u_{0}=0$ and $V_{h}=0$ on $\Gamma$, we obtain

$$
\begin{aligned}
\left|\left\langle\epsilon_{h}, g_{h}\right\rangle\right| & \leqq\left\|\epsilon_{h}\right\|_{L_{2}(\Gamma)}\|g\|_{L_{2}(\Gamma)} \\
& \leqq C h^{\alpha+\sigma / 2-\epsilon}\left[h^{\mu+\sigma / 2-\epsilon}+h^{\sigma}\right]\|f\|_{H^{l}(\Omega)}\left\|\epsilon_{h}\right\|_{L_{2}(\Omega)}, \\
\left|\left\langle\epsilon_{h}, \partial V_{h} / \partial n\right\rangle\right| & \leqq\left\|\epsilon_{h}\right\|_{L_{2}(\Gamma)}\left\|\partial V_{h} / \partial n\right\|_{L_{2}(\Gamma)} \\
& \leqq C\left[h^{\mu+\sigma / 2-\epsilon}+h^{\sigma}\right]\|f\|_{H^{l}(\Omega)}\left\|\epsilon_{h}\right\|_{L_{2}(\Omega)}
\end{aligned}
$$

and

$$
\begin{aligned}
\left|\left\langle\partial u_{0} / \partial n, g_{h}\right\rangle\right| & \leqq C\|f\|_{H^{l}(\Omega)}\left\|g_{h}\right\|_{L_{2}(\Gamma)} \\
& \leqq h^{\kappa+\sigma / 2-\epsilon}\left\|\epsilon_{h}\right\|_{L_{2}(\Omega)}\|f\|_{H^{l}(\Omega)} .
\end{aligned}
$$

Substituting inequalities (4.10)-(4.13) into (4.9), we obtain

$$
\left\|\epsilon_{h}\right\|_{L_{2}(\Omega)} \leqq C(\epsilon)\left[h^{\mu+\kappa-\epsilon}+h^{\kappa+\sigma / 2-\epsilon}+h^{\mu+\sigma / 2-\epsilon}+h^{\sigma}\right]\|f\|_{H^{l}(\Omega)}
$$

and the theorem is proved.

Theorem 4.1 was formulated only for $l$ a nonnegative integer. By using the theory of interpolated spaces, it is easy to get analogous results for $l$ any nonnegative real number.

Institute for Fluid Dynamics and Applied Mathematics

University of Maryland

College Park, Maryland 20742

1. J. H. Bramble \& A. H. Schatz, "On the numerical solution of elliptic boundary value problems by least squares approximation of the data," Numerical Solution of Partial Differential Equations, II (SYNSPADE 1970) (Proc. Sympos., Univ. of Maryland, College Park, Md., 1970), Academic Press, New York, 1971, pp. 107-131. MR 42 \#8713.

2. J. P. Aubin \& H. G. BuRchard, "Some aspects of the method of the hypercircle applied to elliptic variational problems," Numerical Solution of Partial Differential Equations, II (SYNSPADE 1970) (Proc. Sympos., Univ. of Maryland, College Park, Md., 1970), Academic Press, New York, 1971, pp. 1-67. MR 44 \#2359.

3. J. P. AUBIN, Approximations of Elliptic Boundary-Value Problems, Wiley, New York, 1972.

4. I. BabUŠKa, "The finite element method for elliptic differentiation equations," $N u$ merical Solution of Partial Differential Equations, II (SYNSPADE 1970) (Proc. Sympos., Univ. of Maryland, College Park, Md., 1970), Academic Press, New York, 1971, pp. 69-106. MR 42 \#8712.

5. I. BABUŠKa, Numerical Solution of Boundary Value Problems by the Perturbed Variational Principle, Institute for Fluid Dynamics and Appl. Math., Technical Note BN-624, University of Maryland, College Park, Md., 1969.

6. I. BABUŠKA, "The finite element method for elliptic equations with discontinuous coefficients," Computing, v. 5, 1970, pp. 207-213. MR 43 \#2856.

7. J. NiTsCHE, "Über ein Variationsprinzip für die Lösung von Dirichlet Problemen bei Verwendung von Teilräumen, die keinen Randbedingungen unterworfen sind," Abh. Math. Sem. Univ. Hamburg, v. 36, 1970/71.

8. J.-L. LIONS \& E. MAGÈnEs, Problèmes aux Limites non Homogènes et Applications. Vol. 1, Dunod, Paris, 1968. MR 40 \#512.

9. I. BABUŠKA, "Approximation by Hill functions," Comment. Math Univ. Carolinae, v. 11,1970 , pp. 787-811.

10. I. BABUŠKA, "The rate of convergence for the finite element method," SIAM J. Numer. Anal., v. 8, 1971, pp. 304-315. MR 44 \#4918.

11. I. BABUŠKA, "Approximation by Hill functions. II," Comment. Math. Univ. Carolinae, v. 13,1972 , pp. $1-22$.

12. I. BABUŠKA, "Error bounds for finite element method," Numer. Math., v. 16, 1971, pp. 322-333. 